\title{
A study of granular computing in the agenda of growth of artificial neural networks
}

\author{
Mingli Song ${ }^{1}$ Yongbin Wang ${ }^{1}$
}

Received: 20 January 2016/Accepted: 19 April 2016/Published online: 2 May 2016

(C) Springer International Publishing Switzerland 2016

\begin{abstract}
Granular neural networks (GNN) are designed to process complex non-numerical data or the combination of numerical and non-numerical data. The concept of "granules" here refers to various data groups which are drawn together by the criteria of similarity or functionality. Granular neural networks are being used in areas of knowledge discovery, pattern recognition, etc. This paper carries out a comprehensive review of articles that involve a comparative study of different types of granular neural networks and their application. This study aims to give useful insight into the capability of granular neural networks.
\end{abstract}

Keywords Granular neural networks · Granular computing · Information granules · Modular · Fuzzy logic

\section{Introduction}

Artificial neural networks have been widely applied to solve nonlinear and highly adaptable problems. Because of strong nonlinear modeling ability, a traditional neural network has become a useful tool for knowledge discovery and data mining. Unfortunately, a few challenging problems have emerged. One is there is not only numerical data, but also linguistic data such as young, a little bigger and around 3 in various databases. Another is that neural networks are regarded as black boxes owing to the distributed style. As a consequence, they are very difficult to interpret.

Mingli Song

songmingli@cuc.edu.cn

1 School of Computer Science, Communication University of China, Beijing 100024, China
The third problem is that the computational complexity becomes visible as the sizes of the data sets to be handled grow up very quickly. To solve these problems, a new type of neural networks has been proposed, i.e., granular neural networks (with modular structures). The granular neural networks can deal with not only numerical, but alsolinguistic data. They can be interpreted due to the nature of information granules. The property of abstraction decreases the computational complexity. Thus, many researchers are interested in them.

Granular computing is a general computation theory for effectively using granules such as classes, clusters, subsets, groups and intervals to build an efficient computational model for complex applications with huge amounts of data, information and knowledge (Zhang et al. 2000; Zadeh and Kacprzyk 1999; Pal and Meher 2013; Pedrycz et al. 2004; Pedrycz 2002; Bianchi et al. 2014; Pedrycz 2013; Wang and $\mathrm{Xu}$ 2014). Granulation of information can be an effective way of abstraction to solve problems in a hierarchical fashion (Pedrycz and Song 2011). By looking into the main research agenda, we find that granular computing can constitute an ideal conceptual vehicle, supporting further development of neural networks. The ensuing designed networks operating at the level of information granules can be referred to as granular neural networks.

A conceptually viable and practically useful generalization of numeric neural networks comes in the form of nonnumeric mappings realized by neural networks. In this case, it is legitimate to refer to such networks as granular neural networks. The first studies on the combination of fuzzy sets and neural networks are in 1970s (Lee and Lee 1974). Granular neural networks are often used to process linguistic data such as fuzzy terms to do granular knowledge discovery (Zhang et al. 2000). Their different structures can process information granules such as intervals, 
fuzzy sets, rough sets and others by using training algorithms (Pedrycz and Vukovich 2001).

Our main objective is to explore the development of granular neural networks and show how they function. First, the motivation and history of granular neural networks are investigated in Sect. 2. Some preliminaries are given in Sect. 3. Then we illustrate the main categories of granular neural networks and introduce several examples in Sect. 4. A review of granular neural networks' application is given in Sect. 5. Some conclusion ideas are elaborated in Sect. 6 .

\section{Motivation and backgrounds}

Granular neural networks can be a very broad concept in which "granular information" exists in artificial neural networks. The concept of "granular information" here refers to any information that exhibits groups, classes, clusters and linguistic and intervals characteristics. The information can come from input, or structure (includes granular links, granular neurons and granular weights) or output. An intelligent neural network consists of granular neurons and granular links that connect relevant granular neurons. Why is granular information selected? How did they develop? Let us look at our investigation from literature.

\subsection{Why granular?}

Humans often exploit abilities of processing non-numeric information clumps (granules) rather than individual numeric values. Granulation is a suitable way for managing situations characterized by excess or a lack of data. The first situation occurs, for instance, when there are collections of many objects that exhibit some similarity in terms of their properties or functional appearance (Grace 2015). Here, information granulation provides a vehicle to abstract the complexity of the data set that one can organize into hierarchies and convert the original problem into manageable subtasks. The second situation occurs, for instance, when there are noisy data or we encounter qualitative assessments provided by human experts. Here, granulation of information allows modeling the precision of indirect measurements, providing a computationally appealing view of knowledge (Ganivada et al. 2011).

Granular computing is a paradigm of computing with information granules (Yao 2007; Liu et al. 2008). These granules can be regarded as collections of objects that exhibit some similarity in terms of their properties or functional appearance. There are a number of formal models of information granules including sets (interval analysis), rough sets, fuzzy sets, shadowed sets and probability calculus.
In recent years, hybrid neural networks such as fuzzy neural networks, granular neural networks and genetic neural networks have been investigated to solve complex application problems with high dimensionality. However, a single-stage hybrid neural network suffers from the curse of dimensionality, because the number of parameters of the single-stage hybrid neural network increases exponentially with the increasing of the number of inputs. Therefore, to design a powerful granular neural network with high learning speed, low training error and low prediction error is still a challenging problem.

\subsection{Historical developments}

The very first studies about neurons can be traced back to the 1940s (McCulloch and Pitts 1943). Before the birth of the concept of granular neural networks, some related works were discovered and studied. Neuro-fuzzy systems (Pedrycz and Aliev 2009; Lee and Lee 1974; Zhang and Kandel 1998; Frayman et al. 1998; Jang 1993) are a typical example. Neuro-fuzzy systems were first discussed by Lee and Lee (1974) and Kandel and Lee (1979) in the 1970s. Since then, a great many neuro-fuzzy systems have been proposed, with perhaps the best-known being Jang's ANFIS (adaptive network-based fuzzy inference system) architecture (Jang 1993). ANFIS is a fuzzy inference system implemented in the framework of adaptive networks. It implements a Takagi-Sugeno-Kang (TSK) fuzzy system in an adaptive network architecture. Many variations on the ANFIS architecture have been proposed; for instance, almost all learning algorithms for "complex fuzzy logic" systems are variations on ANFIS (Malekzadeh et al. 2004; Chen et al. 2011).

The first thorough study about granular neural networks is Witold Pedrycz's paper (Pedrycz and Vukovich 2001) in around 2000. The paper first introduces the concept of information granules and information granulation which builds the foundation of granular computing. It then elaborates the development process of the granular neural networks which involves two fundamental phases: first, the data (that are usually numeric) are condensed in the form of some information granules and as such are made available to the neural network; second, the resulting information granules are subsequently "perceived" and learned by the neural network and as such used for all training purposes. As a consequence, the neural network is not exposed to the original data of a far higher granularity and far more numerous as the information granules.

Yan-Qing Zhang also proposes a kind of granular neural network for numerical-linguistic data fusion and knowledge discovery in (Zhang et al. 2000) at almost the same time of the paper (Pedrycz and Vukovich 2001). A granular neural network with new high-speed evolutionary interval 
learning is designed to make interval-valued granular reasoning efficiently and optimize interval membership functions based on training data effectively.

\section{Preliminaries}

We often cast a problem into a certain conceptual framework of basic entities, which is regarded to be of relevance to the problem formulation and problem solving. Information granules are an evident realization of the fundamental paradigm of abstraction. In this section, we first give a brief description of several models of information granules used in literature. Then an introduction of commonly used neural networks and the corresponding training algorithms are elaborated.

\subsection{Models of information granules}

We find two taxonomy about the definition of granular data (granular information) in literature. One believes that granular data include various data granules such as classes, clusters, subsets, groups, linguistic values and intervals. The other describes the formal models of information granules as sets (interval analysis), rough sets, fuzzy sets, shadowed sets, probability calculus, etc. From either aspect, information granules can be regarded as an evident realization of the fundamental paradigm of abstraction. From the general point of view, information granules defined in some space $\mathrm{X}$ can be treated as a mapping.

$A: \mathrm{X} \rightarrow \zeta(\mathrm{X})$,

where $A$ is an information granule and $\zeta$ denotes a formal framework of information granules. Some formal models to construct information granules are introduced below.

\subsubsection{Intervals}

Intervals (sets) are examples of sets defined in real lines. The formal underlying model encountered there is also straightforward: a set $A$ is a two-valued mapping from a given universe of discourse $\boldsymbol{X}$ (being a subset of the real

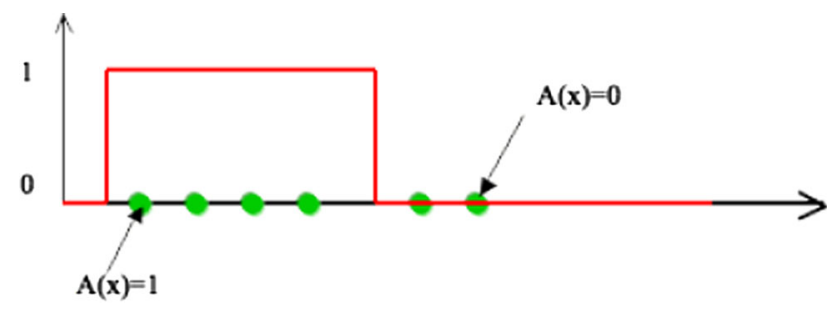

Fig. 1 The concept of "sets" numbers, $\boldsymbol{R})$ to $\{0,1\}$ where $A(x)$ (in Fig. 1) denotes a characteristic function of $A$,

$A: x \rightarrow\{0,1\}$.

Since the nature of straightforwardness and simplicity, intervals have been selected by many users (Cimino et al. 2014; Leite et al. 2013; Pedrycz and Vukovich 2001; Song and Pedrycz 2011, 2013; Zhang et al. 2008) as their frameworks of information granules.

\subsubsection{Fuzzy sets}

Fuzzy sets are generalizations of sets (intervals) in the sense that a partial membership of some elements to the concept represented by a given fuzzy set is admitted. Formally, fuzzy sets are described by their membership functions in Fig. 2,

$A: x \rightarrow 0,1 \geqq$.

Formally speaking, any function could be qualified to serve as a membership function describing the corresponding fuzzy set. The commonly used types are triangular membership functions, trapezoidal membership functions, Gaussian membership functions, etc. (Pedrycz and Gomide 2007). Fuzzy sets are adopted as the granular information in (Zhang et al. 2000; Dick et al. 2013; Sánchez et al. 2015a; Oh et al. 2013; Ganivada et al. 2011; Leite et al. 2013; Pedrycz and Vukovich 2001; Pedrycz et al. 2008; Park et al. 2012; Zhang et al. 2008; Nandedkar and Biswas 2009; Vasilakos and Stathakis 2005; Marcek and Marcek 2008).

\subsubsection{Clusters generated by fuzzy C-means}

Clustering techniques have been regarded as a synonym of structure discovery in data (Pedrycz and Gomide 2007). The result, no matter what technique has been used, comes as a collection of information granules which serve as a quantification of concepts serving as descriptors of the phenomenon behind the data. Fuzzy C-means (FCM) is one of the commonly used mechanisms of fuzzy clustering. The objective function of FCM is defined as:

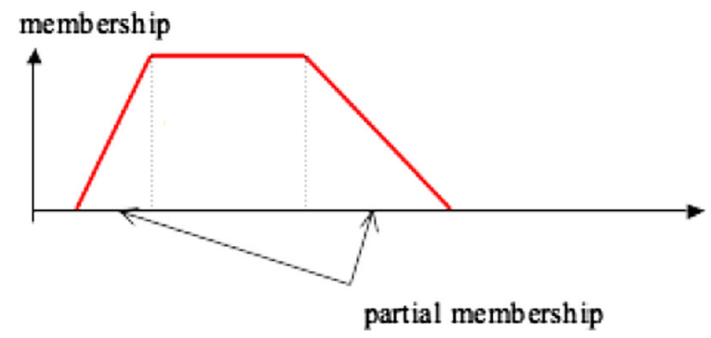

Fig.2 A membership function 
$Q=\sum_{i=1}^{c} \sum_{k=1}^{N} u_{i k}^{m}\left\|x_{k}-v_{i}\right\|^{2}$

The task is to determine the structure in the data represented by a collection of c prototypes $\mathbf{v}_{\mathbf{i}}$, by minimizing $Q$, where $x_{\mathrm{k}}=\left[x_{k 1}, x_{k 2}, \ldots, x_{k n}\right], k=1,2, \ldots, N$, . Patterns are assigned membership values, i.e., a partition matrix $U=\left[u_{i k}\right]$, to the clusters based on their distance from the prototypes. The fuzzification coefficient $m(>0)$ expresses the impact of the membership grades on the individual clusters. The update of the partition matrix is completed as follows:

$u_{i k}(t+1)=\frac{1}{\sum_{j=1}^{c}\left(\frac{\left\|x_{k}-v_{i}(t)\right\|}{\left\|x_{k}-v_{j}(t)\right\|}\right)^{2 /(m-1)}}$.

Some papers choose FCM as a way of generating information granules; please refer to (Park et al. 2009, 2012).

\subsubsection{Clusters generated by context-based fuzzy C-means}

Context-based fuzzy c-means (conditional fuzzy c-means) is a variant of numerous objective-based clustering. The context-based clustering supporting the design of information granules is completed in the space of the input data, while the development of the clusters is guided by a collection of some predefined fuzzy sets (so-called contexts) expressed in the output space (Pedrycz 1996, 1998). The objective function is the same as FCM in (1). However, the update of membership function is changed:

$u_{i k}(t+1)=\frac{s_{i k}}{\sum_{j=1}^{c}\left(\frac{\left\|x_{k}-v_{i}(t)\right\|}{\left\|x_{k}-v_{j}(t)\right\|}\right)^{2 /(m-1)}}$.

Here, in contrast to the matrix formed by the FCM algorithm, $\mathrm{s}_{\mathrm{ik}}$ is not 1.0 but is the $\mathrm{i}$-th row of the partition matrix while $\mathrm{x}$ is the vector of the selected input variables. This exhibits the relationships between the regions of the input space and the output space as partitioning input space based on contexts for information granulation in input space. A family of partition matrices induced by the $j$-th context is denoted by $U\left(T_{j}\right)$ :

$$
\begin{aligned}
U\left(T_{j}\right) & =\left\{u_{i k} \in[0,1], \sum_{i=1}^{c} u_{i k}\right. \\
& \left.=s_{j k} \forall k, 0<\sum_{k=1}^{N} u_{i k}<N \forall i\right\} .
\end{aligned}
$$

The numeric data are granulated with the aid of contextbased fuzzy c-means clustering method in paper (Oh et al. 2013; Pedrycz and Vukovich 2001; Pedrycz et al. 2008; Park et al. 2009).

\subsection{Types of neural networks}

There are mainly two kinds of neural networks commonly used in literature: radial basis function (RBF) and multilayer perceptron (MLP) neural networks. A wide variety of applications, such as chemistry problems (Zupan and Gasteiger 1993), human recognition (Sánchez et al. 2015b) and time series prediction (Gaxiola et al. 2014), among other applications have achieved good results using these neural techniques.

\subsubsection{RBFNN}

An RBF network is an artificial neural network that uses radial basis functions as activation functions. The output of the network is a linear combination of radial basis functions of the inputs and neuron parameters. An RBFNN is a three-layer neural network, in which an $n$-dimensional input vector is transformed in a nonlinear fashion by the receptive fields. Subsequently, the resulting activation levels are transformed in a linear form by the neuron located in the output layer. Quite commonly, the receptive fields are described by the Gaussian basis functions of the form

$\Phi_{i}(x)=\exp \left(-\frac{x-v_{i}^{2}}{2 r_{i}^{2}}\right)$,

where $v_{i}$ and $r_{i}$ are the center (mode) and the width of the Gaussian basis function of the i-th node, respectively. The output of the network $y(x)$ is computed as a linear combination of the individual activation levels, that is

$\mathrm{y}(\mathrm{x})=\sum_{i=1}^{K} w_{i} \Phi_{i}(x)$.

Many people choose RBF neural network as their study. Please refer to (Pedrycz and Vukovich 2001; Pedrycz et al. 2008; Park et al. 2009, 2012; Marcek and Marcek 2008). The general architecture is shown in Fig. 3.

\subsubsection{MLPNN}

A multilayer perceptron is a feedforward artificial neural network model that maps sets of input data onto a set of appropriate outputs. An MLP in Fig. 4 consists of multiple layers of nodes in a directed graph, with each layer fully connected to the next one. In general, MLP utilizes a supervised learning technique called backpropagation for training the network. In granular neural networks, MLP has been used as a basic block (Dick et al. 2013; Cimino et al. 2014; Oh et al. 2013; Ganivada et al. 2011; Song and Pedrycz 2011, 2013). 
Fig. 3 A general architecture of $\mathrm{RBF}$

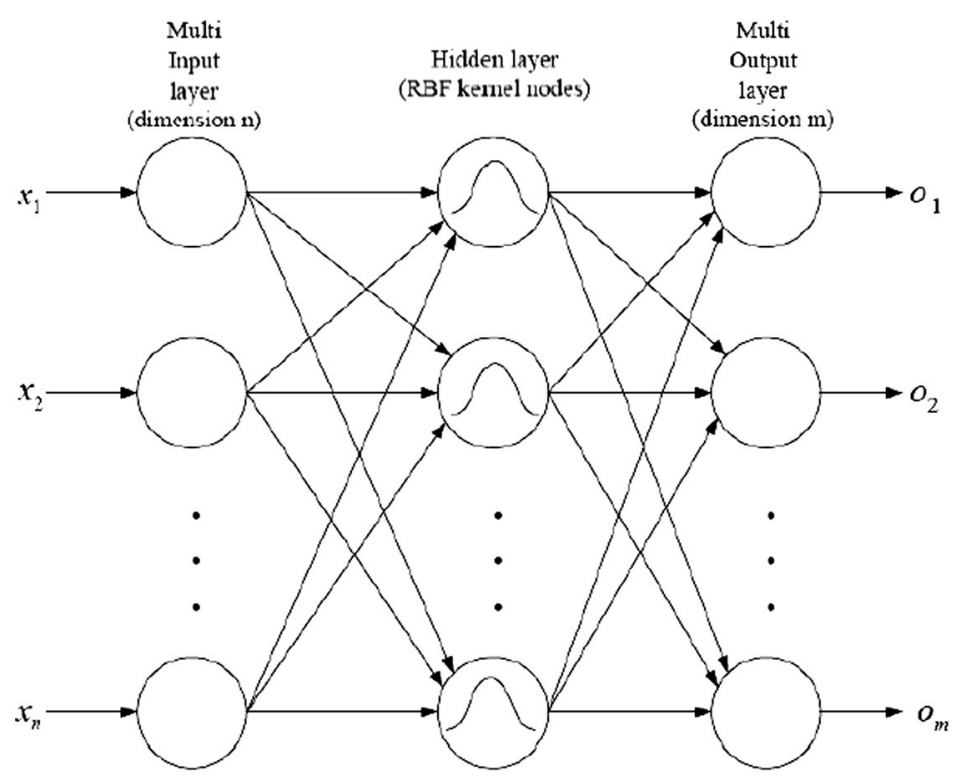

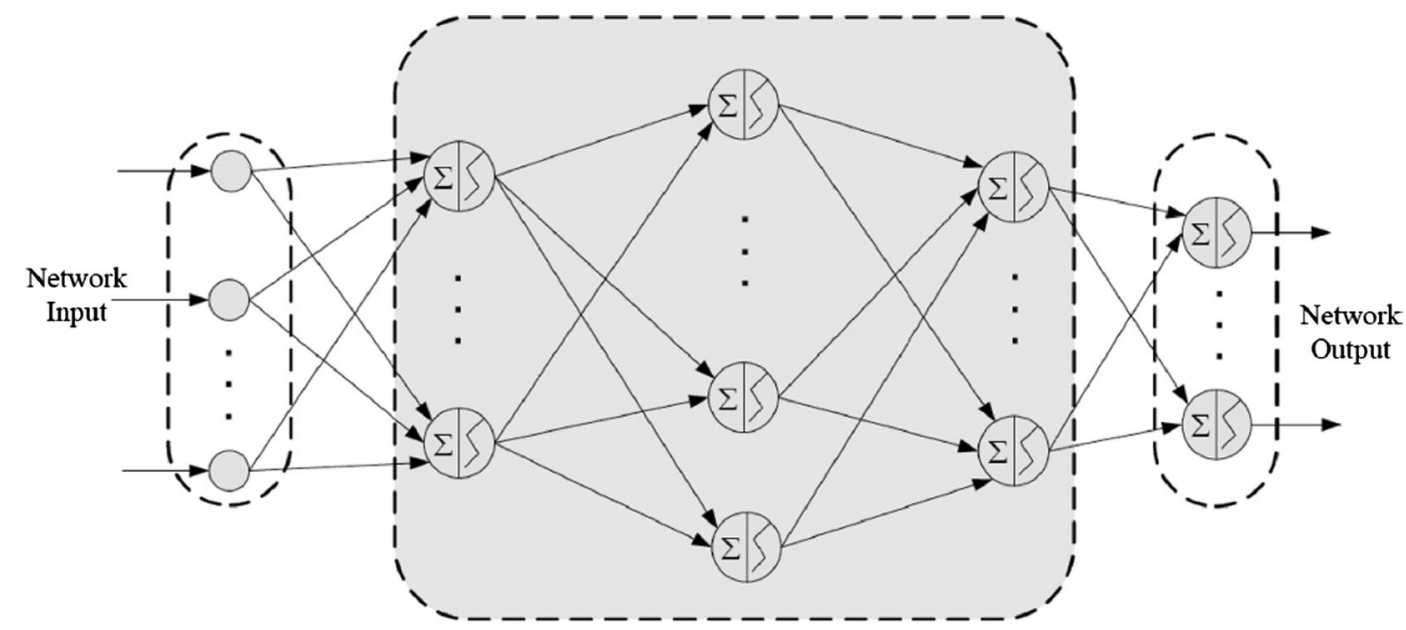

Fig. 4 A general architecture of MLP

Other types of neural networks have been adopted, for instance, multilayer feedforward (MLF) neural networks (Sánchez et al. 2015a).

\subsection{Training algorithms}

A most commonly used training algorithm is backpropagation algorithm which is an abbreviation for "backward propagation of errors". The cumulative error is formulated in (7):

$E_{c}=\sum_{k=1}^{n} E(k)=\frac{1}{2} \sum_{k=1}^{n} \sum_{i=1}^{q}\left(t_{i}(k)-o_{i}(k)\right)^{2}$.

It is a common method of training artificial neural networks used in conjunction with an optimization method such as gradient descent. The method calculates the gradient of a loss function with respect to all the weights in the network. The gradient is fed to the optimization method which in turn uses it to update the weights, in an attempt to minimize the loss function. This algorithm is preferred by many authors (Zhang et al. 2000; Dick et al. 2013; Ganivada et al. 2011; Song and Pedrycz 2013; Vasilakos and Stathakis 2005; Sánchez et al. 2013a).

Other papers have their own choices, for instance, genetic algorithm (Cimino et al. 2014; Sánchez et al. 2015a), the least square estimation (LSE)-based learning (Oh et al. 2013), online incremental learning algorithm (Leite et al. 2013), etc. 


\section{Granular neural networks}

\subsection{Taxonomy of granular neural networks}

The concept of GNN is first formalized in (Pedrycz and Vukovich 2001) by Pedrycz and Vukovich. After some years' development, many different types of GNN have been proposed. These GNN can be categorized in several ways (in Fig. 5). We summarize the taxonomy from three aspects. First, according to the extent of granulation of input, one can put a GNN into four types: training and testing with numeric data $\left(\mathrm{A}_{1}\right)$, training with numeric data but testing with granular data $\left(\mathrm{A}_{2}\right)$, training with granular data and testing with numeric data $\left(\mathrm{A}_{3}\right)$, and training and testing with granular data $\left(\mathrm{A}_{4}\right)$. Depending on whether local data are dealt with separately or together, one can categorize a GNN into a global model with modular models $\left(\mathrm{B}_{1}\right)$ and a model without modular models $\left(\mathrm{B}_{2}\right)$. Another aspect of classification is concerned with a single granular neural network: with granular connection $\left(\mathrm{C}_{1}\right)$ or without $\left(\mathrm{C}_{2}\right)$.

Depending on the extent of granulation of input and architecture, one can categorize GNN into four types as follows (Nandedkar and Biswas 2009) in Fig. 6.

\subsection{1 $A_{1}$ : training and testing with numeric data}

All standard conventional NNs fall under this category. These architectures converge to standard NNs if the data are in the form of numeric quantities.

\subsection{2 $A_{2}$ : training with numeric data and testing on granular data}

This category can be viewed as standard NN with granular connections. A network may be trained using numeric data

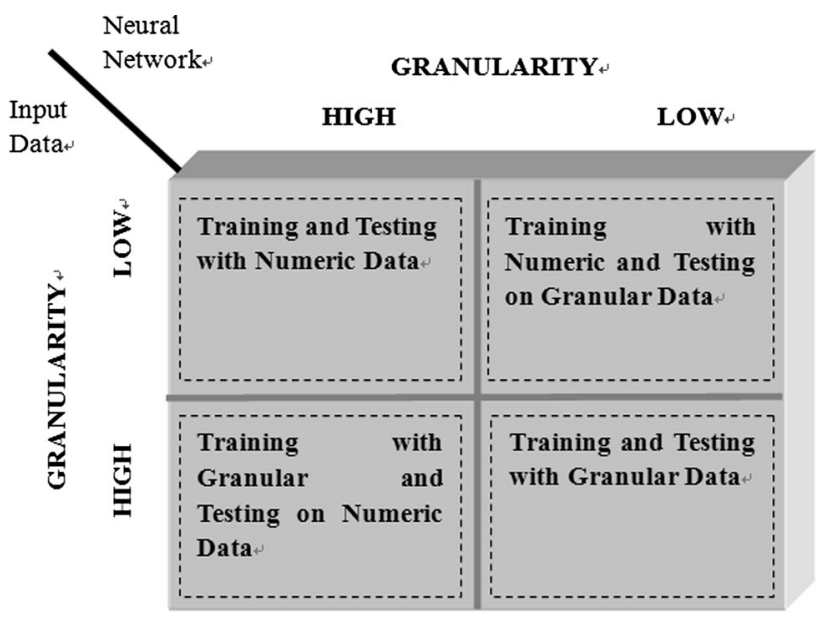

Fig. 6 Four types of GNN

(data of high granularity) and then used in a low-granularity domain. The requirement here is that NN should accept granular data as an input during testing.

\subsection{3 $A_{3}$ : training with granular data and test on numeric data}

There may be a situation where $\mathrm{NN}$ is designed with granular data and needs to operate on high-granularity data. This meansNN structure is of low granularity and current test inputs are of high granularity.

\subsection{4 $A_{4}$ : training and testing with granular data}

The most exigent situation comes forth if the network structure is of low granularity and requires handling input of low granularity. This is the most generalized case of GNN.
Fig. 5 An overall taxonomy of a GNN in literature

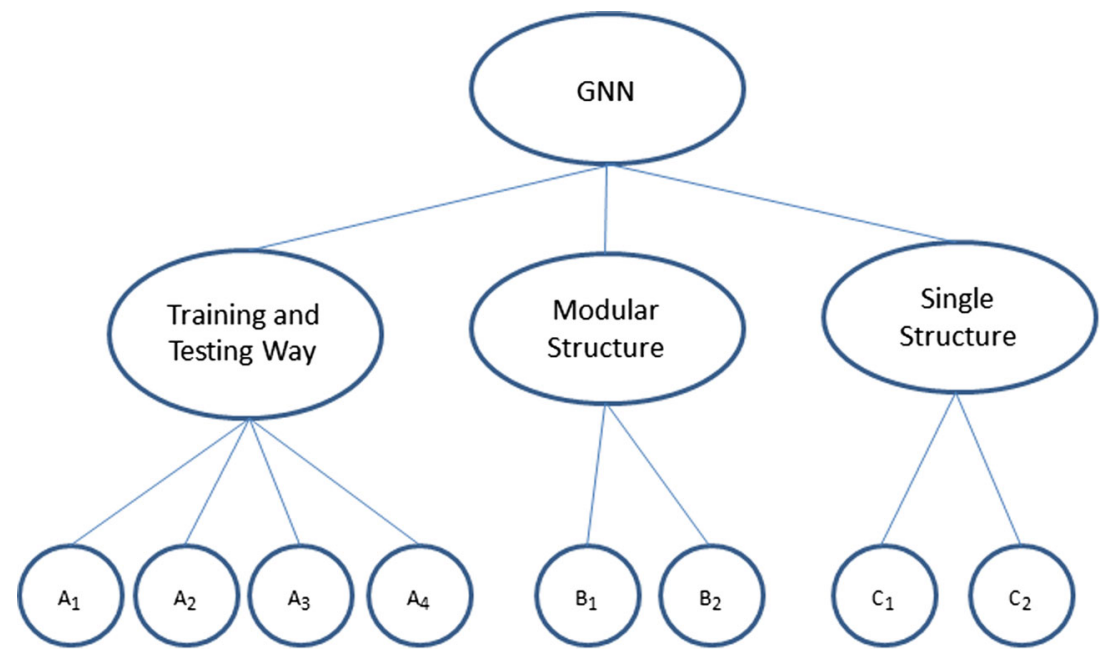


Another interesting taxonomy of granular neural networks is concerned with the way of dealing with the data, local or global. Depending on whether it adopts modular architectures of the neural networks, GNN can be categorized into two types.

\subsection{5 $B_{1}$ : with modular models}

The main difference between conventional neural networks and modular neural networks is that the modular neural networks are an extension of the principle of divide and conquer. This means that the problem can be divided into smaller sub-problems, and these sub-problems are solved by experts, in this case named modules or sub-granules.

Modular neural networks were proposed in (Sánchez and Melin 2014) for human recognition, where a whole database is divided into sub-modules called sub-granules. In this case, each sub-granule can have a different number of data. Also, the information for each phase of the modular neural networks (training and testing phase) is divided with different size in each performance. Granular neural networks which are formed by modular principle with the aid of granular computing techniques have been studied in (Sánchez et al. 2015b; Song and Pedrycz 2011; Sánchez and Melin 2014; Sánchez et al. 2013b).

\subsection{6 $\mathrm{B}_{2}$ : without modular models}

Some granular neural networks prefer single modal from a global perspective. This occurs in some cases in which people focus on dealing with different types of data (Zhang et al. 2000; Cimino et al. 2014; Oh et al. 2013; Ganivada et al. 2011; Leite et al. 2013; Park et al. 2009; Vasilakos and Stathakis 2005).

The architecture of a single granular neural network may have granular connections or numeric connection. Depending on this, we can classify the granular neural networks into two categories:

\subsection{7 $C_{1}$ : with granular connections}

A granular neural network with granular connection means the weights of the network is non-numeric (Song and Pedrycz 2013). In this case, the corresponding training method should also be modified according to the new architecture. When the input data are numeric, the output data generated are non-numeric. When the input data are non-numeric, the output data may be more complicated.

\subsection{8 $C_{2}$ : without granular connections}

Some studies utilize the conventional neural network to deal with complex types of data, such as linguistic terms
(Park et al. 2009; Vasilakos and Stathakis 2005). In this case, researchers only granulate the input data and output data. The values of the weights are still numeric.

\subsection{Selected models of GNN}

In what follows, we choose several representative examples to discuss the details.

\subsubsection{Model $A_{2}$ : training with numeric data and test on granular data}

This type of GNN is trained with numeric data, but can be used for the generation (prediction) of granular data. An example is shown in a paper by Song and Pedrycz (2013). The granular neural network, presented in Fig. 7, includes a single hidden layer with $\mathrm{n}_{1}$ neurons, with one neuron $\mathrm{k}$ in the output layer. The input data are numeric and the output data are intervals. Assume that there are $n$ features of an input variable $\mathbf{x}_{\mathrm{t}}, t=1,2, \ldots, N$. The weight from the ith neuron in the input layer to the $j$ th neuron in the hidden layer is expressed by $W_{j i}$, which is an interval that has two bounds: $w_{j i}^{-}$and $w_{j i}^{+}$. Similar definition applies to weights between hidden layer and output layer. For each neuron in the hidden layer and output layer, there is an individual bias which is represented by $b_{j}$ and $b_{k}$, respectively, $j=1$, $2, \ldots, n_{1}$. In this case, the output of the network is an interval: $y_{t}=\left[y_{t}^{-}, y_{t}^{+}\right]$.

This granular neural network is built from a learned crisp neural network which relies more on the performance

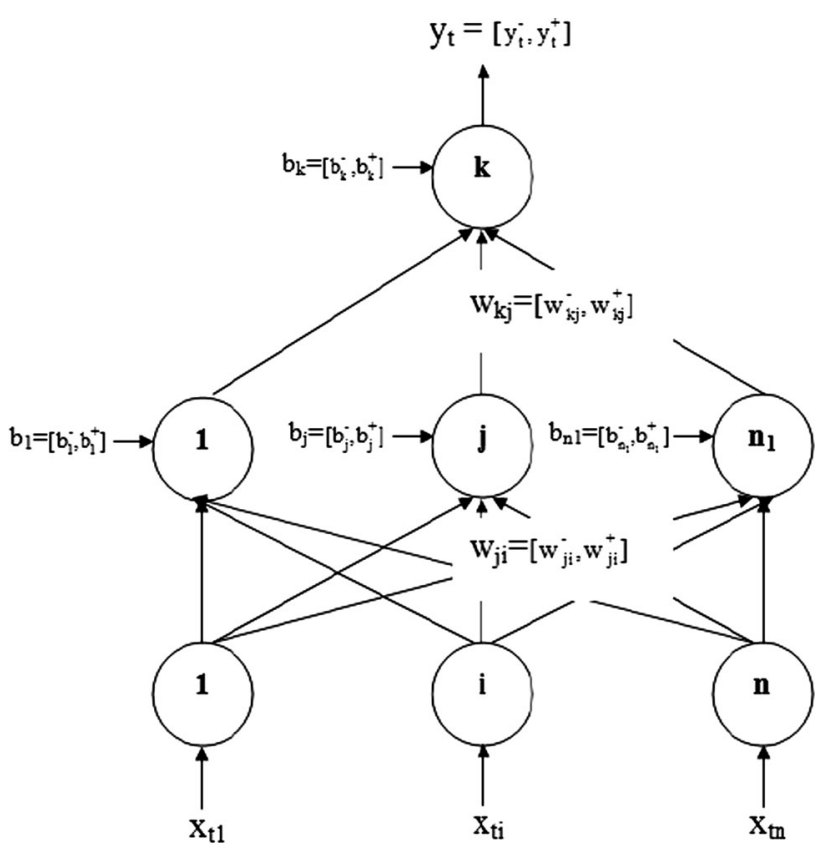

Fig. 7 An architecture of a granular network in Song and Pedrycz (2013) 
of conventional training methods. It is an expansion (or generation) of the conventional neural network. Five protocols are used to build intervals on the weights (connections). It is obvious that the interval-valued connections (weights) of the networks were studied here as one of the simplest alternatives of granular constructs. The two promising generalizations worth pursuing could include fuzzy neural networks and probabilistic neural networks.

\subsubsection{Model $B_{1}$ : with modular structure}

Modular neural networks (MNN) have the main advantage of the principle of divide and conquer. It allows the division of a problem into smaller sub-problems (modules or sub-granules) in Fig. 8. This condition allows achieving better results than with conventional neural networks, because the conditional ones have to learn all the information, unlike in modular neural networks where the information is divided.

The proposed method in the paper (Sánchez et al. 2015b) is based on modular neural networks with a granular approach (Sánchez and Melin 2014) and their optimization based on the database complexity using a hierarchical genetic algorithm. The main idea of this method is to find the optimal architecture of the modular neural networks, because the right architecture depends on the particular application or the database size. This method has two stages: first, the use of non-optimized trainings, and second, based on the results achieved in the first stage, a grouping is performed to obtain the optimal design of the MNN.

For the second stage, a hierarchical genetic algorithm is proposed. The main idea of the optimization is to find out the number of sub-granules or modules. The hierarchical genetic algorithm performs the optimization of the modular neural networks architectures, which consists in finding their optimal parameters. These parameters are the number of modules of each sub-granule, error goal, learning algorithm, number of hidden layers and number of neurons. As

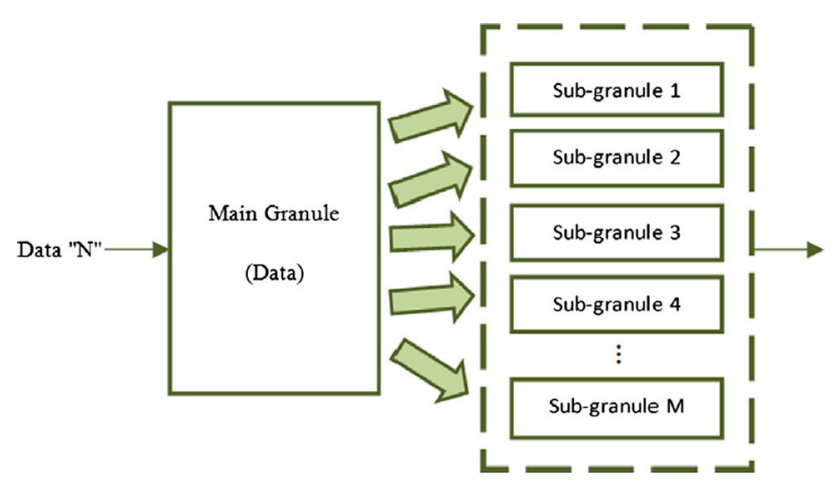

Fig. 8 The general architecture of the proposed method in Sánchez and Melin (2014) future work, the development of a parallel genetic algorithm based on the proposed hierarchical genetic algorithm could be considered, because working with granules and modular neural networks would allow us to perform a division of tasks and therefore to work with parallel genetic algorithms.

\subsubsection{Model $C_{2}$ : without granular connections}

A broad category of neural networks is radial basis function neural networks (RBFNNs) in the sense that its topology involves a collection of receptive fields (Pedrycz et al. 2008; Park et al. 2009). In contrast to the standard architectures encountered in RBFNNs (Park et al. 2009), individual receptive fields in subspaces of the original input space rather than in the entire input space are formed.

These subspaces could be different for different receptive fields. The architecture of the network is fully reflective of the structure encountered in the training data which are granulated with the aid of clustering techniques. More specifically, the output space is granulated with use of $K$ means clustering, while the information granules in the multidimensional input space are formed by using the context-based fuzzy $\mathrm{C}$-means, which takes into account the structure being already formed in the output space. RBFNNs can be treated as three-layered topologies as depicted in Fig. 9.

\subsection{Other ways to construct granular neural networks}

There are some other ways to build a single multilayer perceptron granular neural network in literature (Cimino et al. 2014; Oh et al. 2013; Ganivada et al. 2011; Leite et al. 2013). A granular neural network based on multilayer

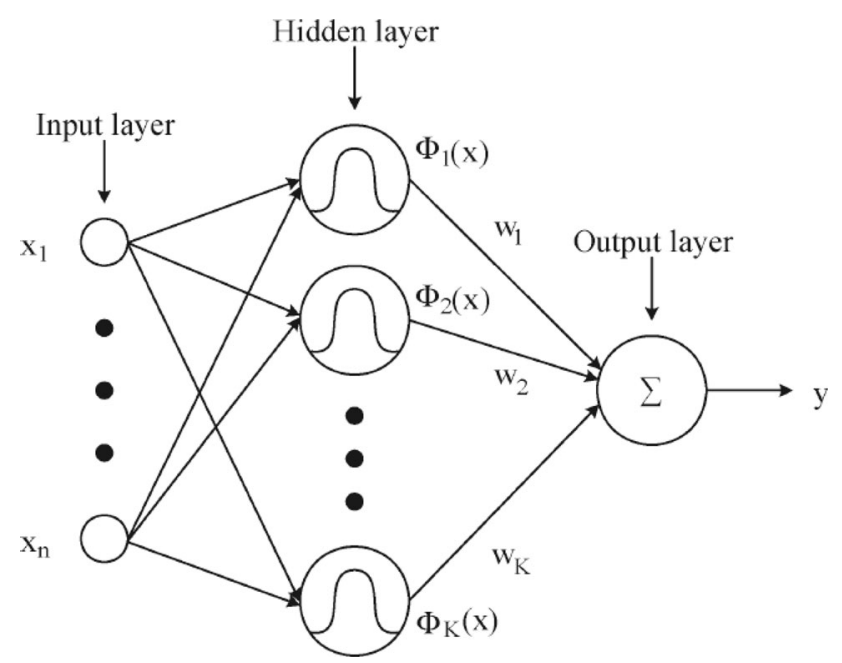

Fig. 9 A general architecture of the RBFNN in Park et al. (2009) 
neural networks for evolving fuzzy system modeling from fuzzy data streams is proposed in (Leite et al. 2013), which is used to handle gradual and abrupt parameter changes typical of nonstationary (online) environments. It combines granular neural networks and evolving intelligent systems concepts into a single modeling approach whose framework aims at coping with four issues: (1) non-interpretability and lack of transparency of black box neural network models; (2) online processing of granular data streams; (3) trading off precision and interpretability; and (4) handling of large volume of data in evolving classification and function approximation problems. The focus is on granular data viewed as trapezoidal fuzzy sets and particular realizations of trapezoids such as triangular fuzzy sets, intervals and real numbers. Ganivada et al. (2011) build a fuzzy rough granular neural network using fuzzy granules as input vector, and fuzzy class membership values and zeros as target vector and multilayer perceptron architecture. The granular network integrates fuzzy rough sets with a fuzzy neural network that uses the dependency factors of all conditional attributes in the form of initial weights of the network and neurons with a logistic activation function and it stores the domain knowledge of the data as a pattern of connection weights between simple processing units. The authors only consider three layers of perceptron network. A multilayer perceptron to model interval-valued input-output mappings named genetic interval neural network is proposed in Cimino et al. (2014). The proposed MLP comes with interval-valued weights and biases and is trained using a genetic algorithm designed to fit data with different levels of granularity. The evolutionary optimization is realized based on a numericvalued and an interval-valued network error.

Some works focus on the construction of granular neural networks using local models (crisp neural networks) (Sánchez et al. 2015a; Song and Pedrycz 2011; Sánchez and Melin 2014). Song and Pedrycz (2011) constructed a granular neural network whose architectures are formed as a direct reconciliation of results produced by a collection of local neural networks constructed on the basis of individual data sets. The authors consider the presence of distributed, individual and locally available data sets generated by the same phenomenon or system being observed from different points of view, which end up with a family of sources of knowledge. In this way, they design a granular neural network-architecture composed of a number of local neural networks whose results are aggregated in the form of information granules. The granularity of information is an inherent manifestation of the diversity of results provided by sources of knowledge. A way of translating numeric data into meaningful information granules is realized through the principle of justifiable granularity. A new model of a modular neural network optimized with hierarchical genetic algorithms is proposed in Sánchez and Melin (2014). A granular approach based on the database complexity is used. Thus, it is called modular granular neural networks. The modular neural networks comprise modules. The design of the architecture of these modular neural networks is performed in two stages. First, non-optimized trainings are obtained, and an initial analysis is performed where labels and new identification numbers (ID) are assigned to each person using if-then rules. Second, the proposed hierarchical genetic algorithm is used, with four different elitism methods, the most important of them called external memory. The proposed method is tested with the problem of human recognition based on the face information.

\section{Trends and applications}

Information explosion is not only creating large amounts of data, but also a diverse set of data. The concept of information granules makes the information more abstract and general. Thus, they inherently become the selection of blocks of system modeling, knowledge discovery and other big "issue". Artificial neural networks are one of them. Although the proposition of the concept of granular neural networks is only about 16 years, its application can be found in many fields. In the following, we will list some cases. Some researchers focus on the foundations and basic theories of granular neural networks (Pedrycz and Vukovich 2001; Zhang 2005; Ding et al. 2014; Zadeh 1998; YZhang 2009; Zhang et al. 2007). On the other hand, people in engineering, industry and other fields like to solve real problems with the aid of granular neural networks (Reformat et al. 2004; García et al. 2008; Saberi et al. 2013; Zhang and Wan 2007; Nandedkar and Biswas 2009; Cruz-Vega et al. 2015; Sánchez and Melin 2013a).

Vasilakos and Stathakis explained land-use classification from remote sensing images by fuzzy neural networks (Vasilakos and Stathakis 2005). Granular neural networks in which fuzzy sets are selected as their formalism framework and an evolutionary training algorithm are introduced in Zhang et al. (2008). A good introduction to neuro-fuzzy inference systems may be found in Jang (1993). One of the earlier neuro-fuzzy systems for classification, named a fuzzy neural network, was developed by Pal and Mitra (Pal and Mitra 1992). As a part of determining the initial weights, Dick and Kandel built a novel neuro-fuzzy system architecture called a linguistic neural network based on information granules to simplify the knowledge representation in the neural networks (Dick and Kandel 2001).

An application of the granular neural network for identifying salient features of data, based on the concepts of fuzzy set and a newly defined fuzzy rough set, is proposed in Ganivada et al. (2013). In this paper, a three- 
layered fuzzy rough granular network is then introduced for feature selection, based on the concept of fuzzy sets and the proposed fuzzy rough set. A pairwise similarity matrix is initially generated by finding the similarity between all possible pairs of patterns, based on fuzzy logical connectives. An $\alpha$-cut is applied on the similarity matrix to develop granulation structures.

Another application of granular neural network named granular-based radial basis function neural networks is to model the charging characteristic of linear-type superconducting power supply (Park et al. 2012). Due to the presence of a large persistent current decay in homogeneity of the HTS coils, a superconducting power supply has been expected to be an important option to maintain the stability of the magnetic field in the superconducting magnet system. The primary aim of the paper is to introduce and design granular-based RBFNNs using clustering methods such as $K$-means clustering and fuzzy $C$-means clustering method and their direct use to modeling of the charging characteristic of linear-type superconducting power supply.

The paper (Marcek and Marcek 2008) extends the application of granular network to fit and predict the quarterly data of wages time series, gives new calculating algorithm for the specific granular network and compares the obtained results with those obtained using statistical procedures. It adopts RBF neural networks architecture. Through the experimental results realized on the quarterly wages time readings of the Slovak economy collected for the period January 1, 1991 to December 31, 2006 which provides a total of 64 observations, the authors conclude that both econometric model and model based on the RBF give approximately identical results.

\section{Summary}

The processing of non-numeric information appears in many scientific fields. It is therefore not surprising to see the evolution of granular computing techniques. Granular neural networks have undoubtedly become one of the most attractive topics due to the powerful function and wide application of neural networks. Granular neural networks have some successful stories in data analysis, system modeling and other fields. In spite of this, machine learning and pattern recognition communities (Maravall and Lope 2011) need to address a number of issues to improve our understanding of granular neural networks. Some problems and research directions are worth focusing in this regard.

(1) The selection of the types of information granules and the granulation processes is the first important issue. There is a profound diversity of the situations that call for information granulation. There is also a panoply of possible formal vehicles to be used to capture the notion of granularity and provide a suitable algorithmic framework in which all granular computing can be efficiently completed.

(2) The learning processes need to be carefully dealt with because of the complexity of the architecture of granular neural networks. No matter how the network is trained, it should reveal the granular architecture of the granular network itself.

Acknowledgments Support from the National Natural Science Foundation of China (NSFC) 61305100, the projects of Communication University of China and Fund of CSCSE are gratefully appreciated.

\section{References}

Bianchi FM et al (2014) A granular computing approach to the design of optimized graph classification systems. Soft Computing 18(2):393-412

Chen Z, Aghakhani S, Man J, Dick S (2011) ANCFIS: a neuro-fuzzy architecture employing complex fuzzy sets. IEEE Trans Fuzzy Syst 19:305-322

Cimino MGCA, Lazzerini B, Marcelloni F et al (2014) Genetic interval neural networks for granular data regression. Inf Sci 257(2):313-330

Cruz-Vega I, Escalante HJ, Reyes CA et al (2015) Surrogate modeling based on an adaptive network and granular computing. Soft Comput. doi:10.1007/s00500-015-1605-9

Dick S, Kandel A (2001) Granular computing in neural networks. In: Pedrycz W (ed) Granular computing: an emerging paradigm. Physica Verlag, New York, pp 275-305

Dick S, Tappenden A, Badke C et al (2013) A granular neural network: performance analysis and application to re-granulation. Int J Approx Reason 54(8):1149-1167

Ding S, Jia H, Chen J (2014) Granular neural networks. Springer Sci 41:373-384

Frayman Y, Wang L (1998) "Data mining using dynamically constructed recurrent fuzzy neural networks," in Proc. PAKDD-98, pp 122-131

Ganivada A, Dutta S, Pal SK (2011) Fuzzy rough granular neural networks, fuzzy granules, and classification. Theor Comput 412(42):5834-5853

Ganivada A, Ray SS, Pal SK (2013) Fuzzy rough sets, and a granular neural network for unsupervised feature selection. Neural Netw 48:91-108

García SR, Romo MP, Botero E (2008) A neurofuzzy system to analyze liquefaction-induced lateral spread. Soil Dyn Earthq Eng 28(3):169-180

Gaxiola F, Melin P, Valdez F, Castillo O (2014) Interval type-2 fuzzy weight adjustment for backpropagation neural networks with application in time series prediction. Inf Sci 260:1-14

Grace A (2015) Signal processing and time series description: a perspective of computational intelligence and granular computing. Appl Soft Computing 27:590-601

Jang J-SR (1993) ANFIS: adaptive-network-based fuzzy inference system. IEEE Trans Syst Man Cybern 23(3):665-685

Kandel A, Lee SC (1979) Fuzzy switching and automata: theory and applications. Crane Russak, New York 
Lee SC, Lee ET (1974) Fuzzy sets and neural networks. J Cybern 4:83-103

Leite D et al (2013) Evolving granular neural networks from fuzzy data streams. Neural Netw 38(2):1-16

Liu S, Wang J, Xing G (2008) The review of outlier mining based on granular computing. In: 2008 IEEE international conference on granular computing, vol 1 and 2. IEEE, New York, USA, pp 462-465

Malekzadeh-AA, Akbarzadeh-TMR (2004) Complex-valued adaptive neuro fuzzy inference system-CANFIS. In: Presented at the Proceedings of World Automation Congress, Seville, Spain

Maravall D, Lope JD (2011) Fusion of learning automata theory and granular inference systems: ANLAGIS. Applications to pattern recognition and machine learning. Neurocomputing 74(8): 1237-1242

Marček M, Marček D (2008) Approximation and prediction of wages based on granular neural network. In: Wang G, Li T, GrzymalaBusse JW, Miao D, Skowron A, Yao Y (eds) Rough sets and knowledge technology, vol 5009. Springer, Berlin, Heidelberg, pp 556-563

McCulloch W, Pitts W (1943) A logical calculus of the ideas immanent in nervous activity. Bull Math Biophys 5:115-133

Nandedkar AV, Biswas PK (2009) A granular reflex fuzzy min-max neural network for classification. Neural Netw IEEE Trans 20(7):1117-1134

Oh S, Kim W, Park B et al (2013) A design of granular-oriented selforganizing hybrid fuzzy polynomial neural networks. Neurocomputing 119(16):292-307

Pal SK, Meher SK (2013) Title paper: natural computing: a problem solving paradigm with granular information processing. Appl Soft Computing 13(9):3944-3955

Pal SK, Mitra S (1992) Multilayer perceptron, fuzzy sets, and classification. IEEE Trans Neural Netw 3(5):683-697

Park HS, Pedrycz W, Oh SK (2009) Granular neural networks and their development through context-based clustering and adjustable dimensionality of receptive fields. Neural Netw IEEE Trans 20(10):1604-1616

Park H, Pedrycz W et al (2012) Modeling of the charging characteristic of linear-type superconducting power supply using granular-based radial basis function neural networks. Expert Syst Appl 39(1):1021-1039

Pedrycz W (1996) Conditional fuzzy c-means. Pattern Recogn Lett 17:625-631

Pedrycz W (1998) Conditional fuzzy clustering in the design of radial basis function neural networks. IEEE Trans Neural Netw 9(4):601-612

Pedrycz W (2002) Computational intelligence and visual computing: an emerging technology for software engineering. Soft Comput $7(1): 33-44$

Pedrycz W (2013) Granular computing as a framework of system modeling. J Control Autom Electr Syst 24(1-2):81-86

Pedrycz W, Aliev RA (2009) Logic-oriented neural networks for fuzzy neurocomputing. Neurocomputing 73:10-23

Pedrycz W, Gomide F (2007) Fuzzy systems engineering: toward human-centriccomputing. Wiley, New York

Pedrycz W, Song M (2011) Analytic hierarchy process (AHP) in group decision-making and its optimization with an allocation of information granularity. IEEE Trans Fuzzy Syst 19(3):527-539

Pedrycz W, Vukovich G (2001) Granular neural networks. Neurocomputing 36:205-224

Pedrycz W, Vasilakos A, Karnouskos S (2004) Guest editorial special issue on computational intelligence in telecommunications networks and internet services-part III. Syst Man Cybern Part C Appl Rev IEEE Trans 34(1):1-3

Pedrycz W, Park HS, Oh SK (2008) A granular-oriented development of functional radial basis function neural networks. Neurocomputing 72:420-435
Reformat M, Pedrycz W, Pizzi N (2004) Building a software experience factory using granular-based models. Fuzzy Sets Syst 145(1):111-139

Saberi M, Mirtalaie MS, Hussain FK et al (2013) A granular computing-based approach to credit scoring modeling. Neurocomputing 122:100-115

Sánchez D, Melin P (2013) Soft computing applications in optimization control and recognition. Springer, Heidelberg 294: 157-185

Sánchez D, Melin P (2013) Multi-objective hierarchical genetic algorithm for modular neural network optimization using a granular approach. In: Castillo O, Melin P, Kacprzyk J (eds) Recent advances on hybrid Intelligent systems, vol 451. Springer, Berlin, Heidelberg, pp 107-120

Sánchez D, Melin P (2014) Optimization of modular granular neural networks using hierarchical genetic algorithms for human recognition using the ear biometric measure. Eng Appl AI 27:41-56

Sánchez D, Melin P, Castillo O, Valdez F (2013) Modular granular neural networks optimization with Multi-Objective Hierarchical Genetic Algorithm for human recognition based on iris biometric. IEEE Congress on Evolutionary Computation 772-778

Sánchez D, Melin P, Castillo O (2015) Optimization of modular granular neural networks using a hierarchical genetic algorithm based on the database complexity applied to human recognition. Inf Sci 309:73-101

Song M, Pedrycz W (2011) From local neural networks to granular neural networks: a study in information granulation. Neurocomputing 74(18):3931-3940

Song M, Pedrycz W (2013) Granular neural networks: concepts and development schemes. Neural Net Learn Syst IEEE Trans 24(4):542-553

Vasilakos A, Stathakis D (2005) Granular neural networks for land use classification. Soft Comput 9(5):332-340

Wang G, Xu J (2014) Granular computing with multiple granular layers for brain big data processing. Brain Inform 1:1-10

Yao J (2007) A ten-year review of granular computing. In: 2007 IEEE international conference on granular computing (GRC 2007). IEEE, Los Alamitos, USA, pp 734-739

Zadeh A (1998) Some reflections on soft computing, granular computing and their roles in the conception, design and utilization of information/intelligent systems. Soft Comput 2(1):23-25

Zadeh LA, Kacprzyk J (eds) (1999) Computing with words in information intelligent systems, vol 1-2. Physica-Verlag, Heidelberg

Zhang Y (2005) Constructive granular systems with universal approximation and fast knowledge discovery. Fuzzy Syst IEEE Trans 13(1):48-57

Zhang Y (2009) Granular neural network, encyclopedia of complexity and systems science, pp 4402-4411

Zhang Y-Q, Kandel A (1998) Compensatory genetic fuzzy neural networks and their applications, series in machine perception artificial intelligenceWorld ScientificSingapore, 30

Zhang YQ, Wan X (2007) Statistical fuzzy interval neural networks for currency exchange rate time series prediction. Appl Soft Computing 7(4):1149-1156

Zhang Y, Gagliano RA et al (2000) Granular neural networks for numerical-linguistic data fusion and knowledge discovery. Neural Netw IEEE Trans 11(3):658-667

Zhang Y, Jin B, Tang Y (2007) Genetic granular neural networks. Adv Neural Netw, pp 1455-1463

Zhang Y et al (2008) Granular neural networks with evolutionary interval learning. Fuzzy Syst IEEE Trans 16(2):309-319

Zupan J, Gasteiger J (1993) Neural networks for chemists. VCH, New York 